\title{
Evaluación de la vascularización del colgajo interoseo posterior mediante angiografía intraoperatoria con indocianina verde
}

\section{Assess of the Posterior Interosseous Flap Vascularization by Indocyanine Green Angiography During Surgery}

\author{
Diego Fernando Gutierrez $^{1}$ Denisse Hartwig ${ }^{1}$ Virginia Giachero ${ }^{1}$ \\ ${ }^{1}$ Cátedra de Cirugía Plástica, Reparadora y Estética, Hospital de \\ Clínicas, Montevideo, Uruguay \\ Address for correspondence Diego Fernando Gutierrez, MD, Hospital \\ de Clínicas Doctor Manuel Quintela, Montevideo, Uruguay \\ Rev Iberam Cir Mano 2019;47:44-47. \\ (e-mail: dfgutierrezm@gmail.com).
}

\section{Resumen}

Palabras clave

- Verde De Indocianina

- procedimientos quirúrgicos reconstructivos

- traumatismos de mano

- colgajos quirúrgicos
La angiografía con indocianina verde es una herramienta que permite, entre otros usos, valorar la vascularización de los colgajos en tiempo real. Presentamos el caso de un paciente con una herida en dorso de mano por extravasación de citostáticos, en el que se plantea una reconstrucción con un colgajo interóseo posterior. La ecografía Doppler preoperatoria no logró identificar la arteria interósea posterior a partir del tercio medio de antebrazo, por lo que realizamos una angiografía con indocianina verde intraoperatoria, que permitió identificar una hipoplasia de la arteria interósea posterior y comprobar la viabilidad del colgajo.

Indocyanine green angiography is a tool that allows, among other uses, to assess the vascularization of the flaps in real time. We report a case of a patient with a wound on the back of his hand due to extravasation of cytostatic, in which a reconstruction with a posterior interosseous flap is considered. The preoperative Doppler ultrasound does not identify the posterior interosseous artery from the middle third of the forearm, so we performed an intraoperative green indocyanine angiography, which allowed us to identify a hypoplasic posterior interosseous artery and asses the viability of the flap.

\section{Introducción}

La indocianina verde (ICG) es un marcador fluorescente, que se une a las proteínas plasmáticas y puede visualizarse mediante una cámara de infrarrojos cercanos (NIR). Actualmente, de acuerdo a la Food and Drug Administration, la angiografía con ICG está indicada para determinar el gasto cardíaco, la función y vascularización hepática y para realizar angiografías

received

May 5, 2018

accepted

September 27, 2018
DOI https://doi.org/

10.1055/s-0038-1675423. ISSN 1698-8396. oftálmicas. ${ }^{1}$ En el área de la cirugía plástica se ha utilizado para: búsqueda del ganglio centinela en melanoma y cáncer de mama, valorar la vitalidad de los colgajos de piel tras la mastectomía, diagnóstico y tratamiento del linfedema y para valorar la vascularización de colgajos libres y pediculados. ${ }^{2,3}$ En este caso, presentamos el uso de la angiografía con ICG durante el procedimiento quirúrgico, para evaluar la
Copyright $\odot 2019$ Thieme Revinter

Publicações Ltda, Rio de Janeiro, Brazil
License terms

(c) $(1) \$$ 


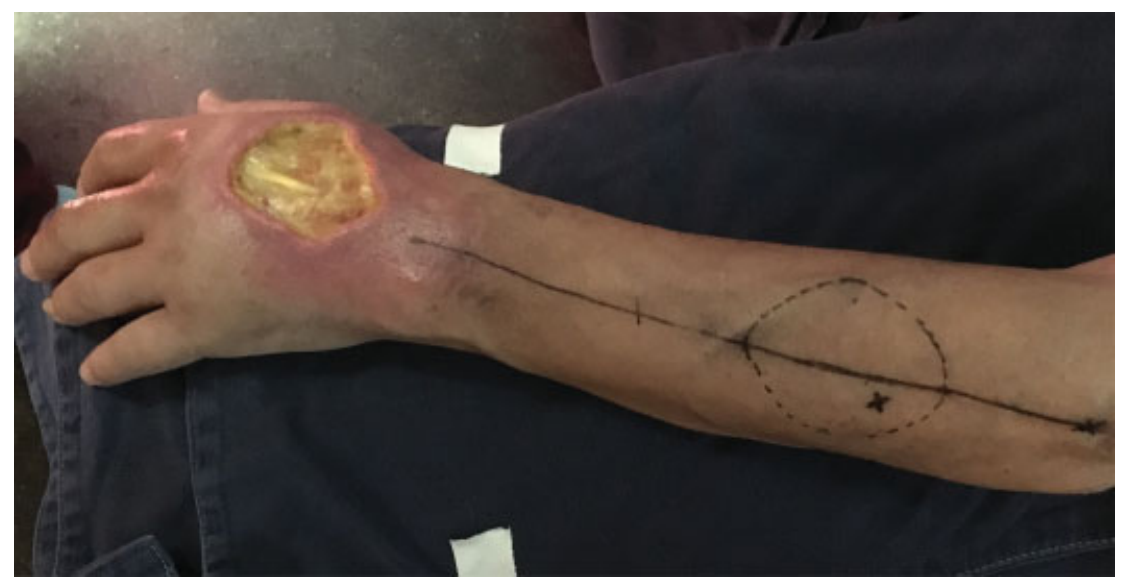

Fig. 1 Foto preoperatoria. Herida en dorso de mano con exposición de tendones del Extensor Digitorum Communis del segundo y tercer dedo. En antebrazo se marcó la perforante y se diseñó el colgajo.

vascularización del colgajo interóseo posterior en un paciente con una variable anatómica de esta arteria.

\section{Caso Clínico}

Varón de 47 años, en poliquimioterapia por un Linfoma No Hodgking retroperitoneal, que sufrió una extravasación de citostáticos en el sitio de punción en dorso de mano, sufriendo una necrosis de la superficie cutánea y exposición de los tendones del Extensor Digitorum Communis del segundo y tercer dedo (-Fig. 1). En la evaluación preoperatoria se solicitó una ecografía Doppler, mediante la cual se identificó y se marcó la arteria perforante nutricia de la isla cutánea. Sin embargo, no se logró identificar la arteria interósea posterior a partir del tercio medio del antebrazo ni la anastomosis distal con la arteria interósea anterior. Por lo tanto, se planteó la realización de una angiografía con ICG durante la cirugía para valorar la viabilidad del colgajo interóseo posterior. Se realizó la cirugía con anestesia general, con el paciente en decúbito supino y el miembro superior en abducción sobre mesa de mano. En primera instancia se realizó el desbridamiento de la herida, resecando los tejidos desvitalizados. La disección del colgajo se realizó bajo isquemia mediante el uso de manguito neumático en brazo, que se retiró al momento de la angiografíia. Se diseñó la isla cutánea del colgajo de acuerdo al defecto, midiendo esta $6 \times 8 \mathrm{~cm}$. El abordaje fue sobre una línea que une el epicóndilo humeral a la articulación radiocubital distal. Mediante magnificación con lupas 2x, se disecó de radial a cubital el colgajo y su perforante cutánea. A ese nivel, se abordó longitudinalmente la aponeurosis profunda, identificando la arteria interósea posterior en su sector proximal. El calibre de la arteria se estrechaba después de las primeras comunicantes y parecía discontinuarse. A continuación, se vació el manguito neumático para realizar la angiografía con ICG. Se administraron 3 bolos de ICG 99mTc nanocoloide por vía venosa periférica. El primero permitió distinguir el sector distal de la arteria interósea posterior, donde era difícil de disecar sin el contraste. Los siguientes bolos de ICG se introdujeron al elevar el colgajo y al transponerlo sobre la herida (-Figs. 2A, B y C). En ambos casos el colgajo se tiñó completamente con ICG, evidenciando su buena irrigación a pesar de la manipulación y movilización del pedículo. El colgajo se transpuso hacia el defecto y se suturó a los bordes del defecto. Se procedió a la cobertura del área dadora cutánea y de parte del pedículo con un injerto de piel parcial tomado del muslo.
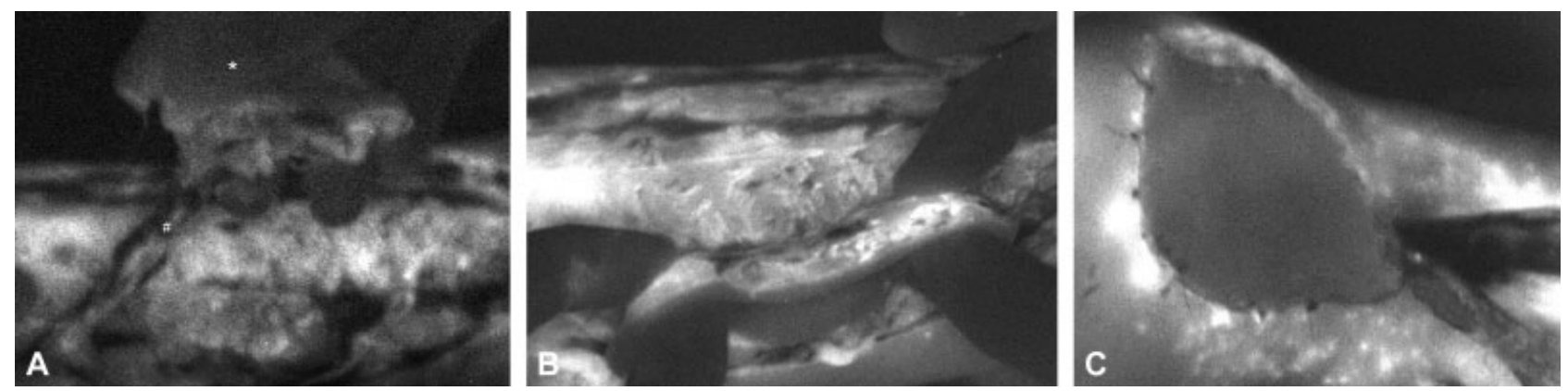

Fig. 2 Imágenes intraoperatorias obtenidas con la cámara NIR: (A) El * señala el colgajo en la mano del cirujano, y el \# marca el pedículo, donde se ve la arteria teñida de blanco; (B) Muestra el colgajo después del segundo bolo, ya que el tejido subcutáneo se tiñe de forma más intensa que la piel; (C) El colgajo cubriendo la herida de mano con una vascularización adecuada, el tejido circundante se encuentra más perfundido debido a la inflamación e hiperemia que presenta. 


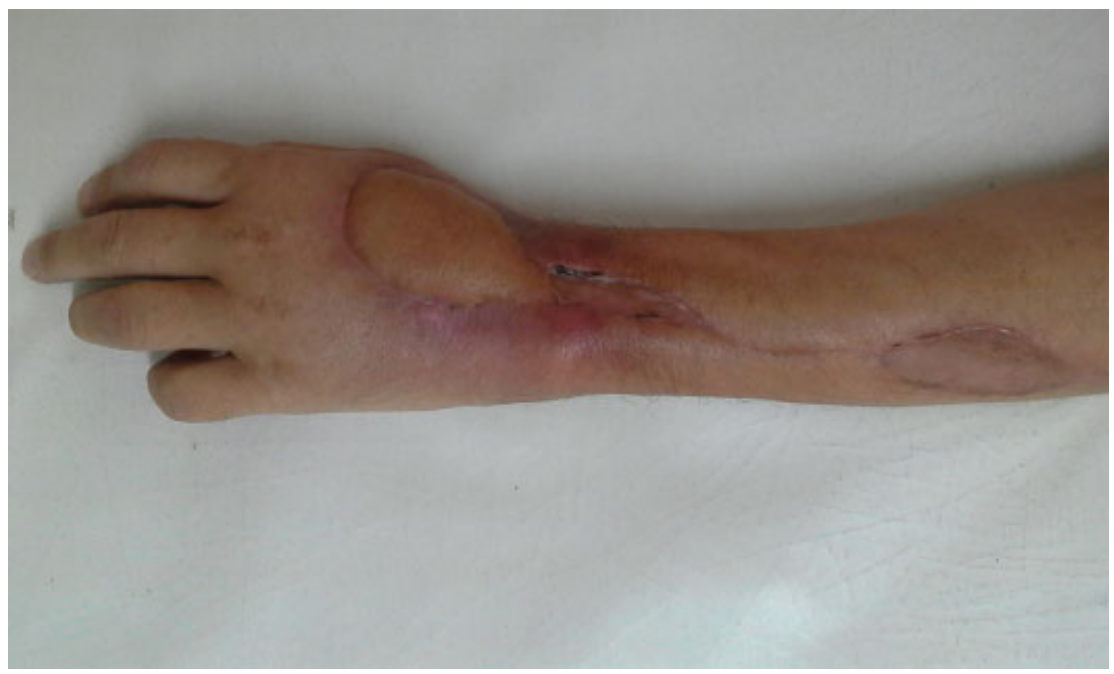

Fig. 3 Evolución luego de 1 mes postoperatorio.

El período postoperatorio transcurrió sin complicaciones (-Fig. 3). Se mantuvo la muñeca inmovilizada con una férula antebraquio palmar durante dos semanas, con movilización de los dedos desde el primer día. Acudió a la rehabilitación con una evolución satisfactoria, continuando con su tratamiento oncológico. A los 4 meses sufrió una recaída de su enfermedad y falleció.

\section{Discusión}

Desde que Zancolli y Angrigiani describieron por primera vez el colgajo interoseo posterior en $1986,{ }^{4}$ son muchos los estudios que han demostrado que es una alternativa reproducible, fiable y efectiva para la cobertura del dorso de mano hasta la primer falange del pulgar y el primer espacio interdigital. ${ }^{5-7}$ Una desventaja de ese colgajo son las posibles variaciones anatómicas de la arteria interósea posterior. Se describen 2 variaciones anatómicas principales: la ausencia de la anastomosis distal con la arteria interósea anterior, y la hipoplasia o aplasia del tercio medio de la arteria interósea posteior. ${ }^{5}$ Penteado y col. ${ }^{6}$ encontraron estas variaciones anatómicas en 5 de 70 disecciones anatómicas, siendo: 4 aplasias o hipoplasias del tercio medio de la arteria interosea posterior, y 1 ausencia de la anastomosis distal. Angrigiani y col., ${ }^{7}$ en un estudio de 80 disecciones anatómicas y 80 casos clínicos, describen 2 casos de discontinuidad de la arteria interósea posterior en su tercio medio, que corresponden a una disección anatómica y un caso clínico. Además, presentan que en 74 de las 80 disecciones, la arteria interósea posterior se afina en su tercio medio, donde tiene un diámetro menor que en el sector distal.

Costa y col., ${ }^{5}$ presentaron un estudio con 100 disecciones anatómicas y 81 pacientes, en el que no encuentra variaciones anatómicas en las disecciones cadavéricas, pero en 3 casos clínicos refiere que la arteria parecía discontinuarse en el tercio medio del antebrazo por lo cual realizaron otros métodos de reconstrucción alternativos. Otros casos clínicos describen el hallazgo intraoperatorio de una variedad anatómica de la arteria interósea posterior requiriendo usarlo como colgajo libre o realizar otro tipo de colgajo. ${ }^{8,9}$ Para evitar este problema, distintos autores recomiendan realizar un Doppler o una ecografía Doppler preoperatoria para descartar variaciones anatómicas. ${ }^{8-10}$ En nuestra experiencia, la ecografía Doppler preoperatoria tiene gran utilidad para la valoración del pedículo vascular del colgajo. En el caso clínico presentado, el Doppler no pudo determinar la permeabilidad del pedículo, sospechándose una posible variable anatómica. Se decidió realizar una angiografía con ICG intraoperatoria la cual nos permitió identificar un pedículo vascular hipoplásico pero con buena vascularización del colgajo. Probablemente sin esa herramienta y siguiendo la experiencia de otros autores, se hubiera descartado la realización del colgajo interóseo posterior, realizándose otro tipo de cobertura. La angiografía con ICG en el intraoperatorio permite una evaluación precisa y en tiempo real sobre la vascularización del colgajo, y además marca el territorio de piel que corresponde a la perforante. ${ }^{2}$ Eso último posiblemente puede disminuir la incidencia de pérdidas parciales del colgajo interóseo posterior, descritas en las series de casos. ${ }^{10}$

Dado que la ICG presenta un corto tiempo de vida media (35 minutos) y un amplio margen de seguridad, se pueden administrar varios bolos durante el procedimiento. ${ }^{3}$ Eso nos permitió comprobar la vascularización del colgajo en distintos tiempos de la cirugía. De acuerdo a la evolución del paciente con la técnica realizada, sugiere que la angiografía con ICG intraoperatoria es una herramienta fiable y útil para valorar la viabilidad del colgajo interóseo posterior. Sin embargo, es necesario un estudio con mayor número de pacientes para determinar su verdadero valor para ese fin.

\section{Bibliografía}

1 U.S. Food \& Drug Administration. IC-Green. Highlights of prescribing information. Disponible en: https://www.accessdata.fda.gov/drugs atfda_docs/label/2015/011525s027lbl.pdf. Fecha de acceso: 16/04/ 2018

2 Liu DZ, Mathes DW, Zenn MR, Neligan PC. The application of indocyanine green fluorescence angiography in plastic surgery. J Reconstr Microsurg 2011;27(06):355-364 
3 Burnier P, Niddam J, Bosc R, Hersant B, Meningaud JP. Indocyanine green applications in plastic surgery: A review of the literature. J Plast Reconstr Aesthet Surg 2017;70(06):814-827

4 Zancolli EA, Angrigiani C. Colgajo dorsal de antebrazo en isla (Pedículo de vasos interóseos posteriores). Rev Asoc Orthop Traumatol 1986;54:161-168

5 Costa H, Gracia ML, Vranchx J, Cunha C, Conde A, Soutar D. The posterior interosseous flap: a review of 81 clinical cases and 100 anatomical dissections-assessment of its indications in reconstruction of hand defects. Br J Plast Surg 2001;54(01):28-33

6 Penteado CV, Masquelet AC, Chevrel JP. The anatomic basis of the fascio-cutaneous flap of the posterior interosseous artery. Surg Radiol Anat 1986;8(04):209-215
7 Angrigiani C, Grilli D, Dominikow D, Zancolli EA. Posterior interosseous reverse forearm flap: experience with 80 consecutive cases. Plast Reconstr Surg 1993;92(02):285-293

8 Pauchot J, Lepage D, Leclerc G, Flamans B, Obert L, Tropet Y. Lambeau interosseux postérieur libre pour absence d'artère interosseuse postérieure. Une adaptation technique a une variation anatomique rare. Cas clinique et revue de la littérature. Ann Chir Plast Esthet 2010; 55(01):56-60

9 Giunta R, Lukas B. Impossible harvest of the posterior interosseous artery flap: a report of an individualised salvage procedure. Br J Plast Surg 1998;51(08):642-645

10 Büchler U, Frey HP. Retrograde posterior interosseous flap. J Hand Surg Am 1991;16(02):283-292 